\title{
Genetic Bias in Immune Responses to a Cassette Shared by Different Microorganisms in Patients with Rheumatoid Arthritis
}

\author{
Antonio La Cava, ${ }^{\S}$ J. Lee Nelson, ${ }^{ף}$ William E.R. Ollier, ${ }^{\star \star}$ Alexander MacGregor, ${ }^{\ddagger}$ Edward C. Keystone, ${ }^{\S \S}$ J. Carter Thorne, ${ }^{\S \S}$ \\ John F. Scavulli, $\mid \|$ Charles C. Berry, $\|$ Dennis A. Carson, ${ }^{\star \S}$ and Salvatore Albani ${ }^{\star} \ddagger \S$ \\ $*$ Department of Medicine, ${ }^{\ddagger}$ Department of Pediatrics, ${ }^{\circledR}$ The Sam and Rose Stein Institute for Research on Aging, and $\|_{\text {the Department of }}$ \\ Family and Preventive Medicine, University of California, San Diego, La Jolla, California 92093-0663; "Division of Immunogenetics, Fred \\ Hutchinson Cancer Research Center, Seattle, Washington 98104; **ARC Epidemiology Research Unit, University of Manchester, \\ Manchester, M13 9PT, United Kingdom; ${ }^{\ddagger}$ Twin Research Unit, St. Thomas Hospital, London, United Kingdom; ${ }^{\S}$ Rheumatic Diseases \\ Unit, Wellesley Hospital, Toronto, Ontario M4Y 1J3, Canada; and ${ }^{\| l}$ Kaiser Permanente Hospital, San Diego, California 92120
}

\begin{abstract}
Rheumatoid arthritis (RA) is an autoimmune disease associated with HLA-DR $\beta 1$ alleles which contain the QKRAA amino acid sequence in their third hypervariable region(s). The QKRAA sequence is also expressed by several human pathogens. We have shown previously that an Escherichia coli peptide encompassing QKRAA is a target of immune responses in RA patients. Here we address two questions: first, whether QKRAA may function as an "immunological cassette" with similar, RA-associated, immunogenic properties when expressed by other common human pathogens; and second, what is the influence of genetic background in the generation of these responses. We find that early RA patients have enhanced humoral and cellular immune responses to Epstein-Barr virus and Brucella ovis and Lactobacillus lactis antigens which contain the QKRAA sequence. These results suggest that the QKRAA sequence is an antigenic epitope on several different microbial proteins, and that RA patients recognize the immunological cassette on different backgrounds. ANOVA of immune responses to "shared epitope" antigens in monozygotic twin couples shows that, despite significantly elevated responses in affected individuals, a similarity between pairs is retained, thus suggesting a role played either by hereditary or shared environmental factors in the genesis or maintenance of these responses. (J. Clin. Invest. 1997. 100:658-663.) Key words: autoimmunity - rheumatoid arthritis - molecular mimicry - HLA • shared epitope
\end{abstract}

\section{Introduction}

Rheumatoid arthritis (RA) is strongly associated with HLADR alleles containing, in the third hypervariable region of their $\beta$ chains, the QK/RRAA (glutamine-lysine/arginine-arginine-alanine-alanine) amino acid motif (1-4). How this "shared epitope" (5) increases RA severity or susceptibility is still controversial. We have shown previously that patients

Address correspondence to Dr. Antonio La Cava, University of California, San Diego, 9500 Gilman Drive, La Jolla, CA 92093-0663. Phone: 619-534-5392; FAX: 619-534-5399; E-mail: antonio@ucsd.edu

Received for publication 24 October 1996 and accepted in revised form 22 April 1997.

J. Clin. Invest.

(C) The American Society for Clinical Investigation, Inc. 0021-9738/97/08/0658/06 \$2.00

Volume 100, Number 3, August 1997, 658-663

http://www.jci.org with early RA have abnormal cellular and humoral immune responses to the QKRAA sequence derived from the Escherichia coli dnaJ heat shock protein (6). We have proposed a model for RA, in which $\mathrm{T}$ cells, positively selected in thymus by low-affinity interactions with self-MHC peptides, may be later triggered in the periphery, upon exposure to foreign peptides similar enough to cross-react and to break immunological tolerance $(6,7)$. Similar mechanisms might occur for other autoimmune diseases associated with certain HLA alleles $(8,9)$.

The model takes in consideration the following aspects of RA pathogenesis: $(a)$ the disease has a genetic substrate; $(b)$ environmental factors probably play an important role in disease initiation; $(c)$ there is no epidemiological evidence linking RA to specific environmental stimuli; and $(d)$ with few exceptions, no geographical prevalence of the disease is observed. If microbial agents are involved in RA etiopathogenesis because they carry the QKRAA susceptibility sequence to RA (10), these microorganisms should be common enough to infect asymptomatically a large number of people. E. coli fulfills these requirements, and its dnaJ heat shock protein, which contains QKRAA, has been shown to be a target of specific responses in RA patients (6). Another possibility to explain the lack of epidemiological associations in RA is that the relevant antigen is shared by more than one microorganism. More recently, other common mucosal pathogens have been described which contain the QKRAA sequence (11-15). Therefore, it was important to ascertain whether the exogenously derived QKRAA sequence might be immunologically relevant for RA when expressed by microorganisms different from $E$. coli. Here, we analyzed the immune responses of early RA patients to peptides and proteins from Epstein-Barr virus (EBV), Lactobacillus lactis, and Brucella ovis, which express the QKRAA sequence. The results show that shared epitope positive RA patients respond more strongly than normal subjects to these antigens.

To evaluate the role played by genetics in the generation and/or perpetuation of these abnormal responses, we also analyzed immune reactivity to four recombinant proteins encompassing the shared epitope in 22 monozygotic twin pairs discordant for RA. ANOVA shows that either genetics or exposure to common environmental agents contributes to the immune recognition of antigens encompassing the QKRAA sequence. However, significantly increased responses to the shared epitope are found only in the RA-affected twins, further supporting the concept that these responses are associated with the disease.

\section{Methods}

Patients. 108 patients with RA were studied. Diagnosis was performed according to the American College of Rheumatology criteria 
Table I. Clinical Characteristics of SE Positive Patients with Early $R A(n=25)$

\begin{tabular}{ll}
\hline Age & $56.6 \pm 19.3 \mathrm{yr}$ \\
Sex & 8 males, 17 females \\
$\begin{array}{l}\text { Disease duration } \\
\text { Number of affected }\end{array}$ & $7.1 \pm 5.5 \mathrm{mo}$ \\
$\quad$ joints $(n=21)$ & $15.6 \pm 9.7$ \\
$\begin{array}{l}\text { Erythrocyte sedimen- } \\
\text { tation rate }(n=21)\end{array}$ & $56.1 \pm 63.4 \mathrm{~mm} / \mathrm{h}$ \\
Rheumatoid factor & $\begin{array}{l}15 \text { positive, } 10 \text { negative } \\
\text { Therapy }\end{array}$ \\
$\begin{array}{l}24 \text { patients were taking nonsteroidal anti- } \\
\text { inflammatory drugs; } 2 \text { were also } \\
\text { receiving hydroxychloroquine, } 1 \text { was } \\
\end{array}$ & receiving no therapy.
\end{tabular}

(16). 25 shared epitope (SE) ${ }^{1}$ positive patients with early RA $(7.1 \pm 5.5 \mathrm{mo})$ were studied for T cell responses. Diagnosis for most of these patients was retroactive. Clinical characteristics for the 25 patients are given in Table I. As a control, 6 SE negative RA patients, as well as 14 healthy individuals typed as HLA-DRB1*0401 or *0404, were included in $\mathrm{T}$ cell proliferation assays. Control subjects for humoral studies included 10 SE negative RA patients, 56 HLA-DRB matched healthy individuals, and 15 patients with SLE. We also used sera from 22 monozygotic twin pairs, all HLA-DR4 positive.

Antigens. We used as recombinant antigens gp110 glycoprotein (858 aa), encoded by the BALF4 open reading frame of EBV, and two gp110 deletion mutants, a carboxy-terminal fragment of 281 amino acids containing the QKRAA sequence, and an amino-terminal fragment of 347 amino acids not containing QKRAA. Recombinant antigen expression and purification were performed as described elsewhere (La Cava, A., A. Martini, D.A. Carson, and S. Albani, manuscript submitted for publication). Briefly, DNA from EBV strain B95-8 was used as template for PCR amplification of gp110 glycoprotein (using the primers 1: 5'GCCCAAGCTTGGGATGACTCGGCGTAGGGTG3' and 2: 5'CGGGATCCCGCTATTAAAACTCAGTCTCTGC3') and of the two deletion mutants, one including the QKRAA sequence (primers 1 and 3: 5'CGGGATCCCGCTACTCATGCATGGTCTTGTT3') and one not (primer 4: 5'GACAACGAGATCTTCCTCACAAAAAAG3' and 2). PCR conditions were as follows: hot start at $95^{\circ} \mathrm{C}$ for $5^{\prime}$, first 2 cycles annealing at $55^{\circ} \mathrm{C}, 32$ cycles annealing at $58^{\circ} \mathrm{C}$, last cycle $8^{\prime}$ extension. The restriction sites for BamHI and HindIII contained in the primers were used for subcloning into the prokaryotic vector pSE420 (Invitrogen Corp., San Diego, CA) and into the eukaryotic vector p290 (kindly provided by Dr. Tyler Parr). After bacterial transformation with the pSE420-gp110/deletion mutants constructs, recombinant protein expression was induced with $1 \mathrm{mM}$ isopropylthiogalactoside. Recombinant proteins were purified from the bacterial lysates by passage through immunoaffinity columns. Anti-gp110/anti-deletion mutant antibodies for immunoaffinity columns were obtained by injecting intradermally $400 \mu \mathrm{g}$ of a naked DNA eukaryotic plasmid expression vector encoding the p290-gp110/deletion mutants into New Zealand White rabbits, four times, at weekly intervals. Generation of specific antibodies was monitored weekly by Western blotting, using as antigen the bacterial lysates. Hyperimmune $\operatorname{IgGs}$ were purified on protein A columns (Pierce, Rockville, MD) and covalently bound to agarose-hydrazide solid supports (Affi-Gel, Bio-Rad Laboratories, Hercules, CA). All purified antigens produced a single ma-

1. Abbreviation used in this paper: SE, shared epitope. jor band of the expected molecular weight after denaturing polyacrylamide gel electrophoresis and staining by Coomassie blue.

Synthetic peptides were purchased from the Molecular Biology Core of the Research Institute of Scripps Clinic (La Jolla, CA), and were $>90 \%$ pure, as determined by HPLC. The sequences of the peptides are as follows: gp110-p: QKRAAQRAAGPSVAS; bo-p: QKRAAYDRFGHAAFE; ll-p: QKRAAYDQYGEAGAN; dnaJp1: QKRAAYDQYGHAAFE; self-p: QKRAAVDTYCRHNYG. Gp110-p encompasses the region of homology (QKRAA) of gp110 (amino acid 808-812) with HLA-DRB1*0401 (11). Bo-p, ll-p, and dnaJ-p1 derive their sequences from B. ovis, L. lactis and E. coli dnaJ heat shock proteins, respectively, and also encompass the QKRAA motif (12-15). Self-p is an HLA-DRB1*0401-derived peptide including the QKRAA sequence.

HLA peptide binding assay. This assay has been described previously $(6,17)$. Briefly, lymphoblastoid cell lines generated from an HLA-DRB1*0401 RA patient, an HLA-DR*0401 healthy control, and from a DRB1*0701 control were used as sources of HLA molecules.

EIA/RIA plates (Costar Corp., Cambridge, MA) were coated with $40 \mu \mathrm{g} \mathrm{ml}^{-1}$ of anti-HLA class II IgG obtained from HB145 hybridoma (American Type Culture Collection, Rockville, MD). After washing and blocking, HLA class II molecules were added at a concentration of $10 \mu \mathrm{g} \mathrm{ml}^{-1}$. After $2 \mathrm{~h}$, plates were washed and incubated with $10 \mathrm{nM}$ of biotinylated peptides, previously labeled with an ImmunoProbe Biotinylation Kit (Sigma Chemical Co., St. Louis, MO). After washing and incubation with alkaline phosphatase-conjugated ExtraAvidin, activity was assessed colorimetrically. Tests were performed in duplicate. $50 \%$ inhibition was achieved at a peptide concentration of $100 \mathrm{nM} \mathrm{ml}^{-1}$. Results are expressed as OD values at $405 \mathrm{~nm}$.

ELISA. Antibodies against the bacterial antigens were quantitated by ELISA at serum dilutions of 1-100, as described (6). Results are expressed as 100 times the average OD at $405 \mathrm{~nm}$ of duplicate wells for the test sera, divided by the OD of a standard reference serum included in each assay. Inhibition studies were performed by preincubating sera with $100 \mu \mathrm{g} \mathrm{ml}^{-1}$ peptide or with irrelevant control peptide overnight at room temperature, before performance of the ELISA.

Detection of previous EBV infection. Previous EBV infection was assessed for patients and matched control sera using the Pharmacia EBV IgG ELISA kit (Pharmacia Biotech, Piscataway, NJ).

Lymphocyte proliferation assays. Peripheral blood mononuclear cells were stored in liquid $\mathrm{N}_{2}$ at a density of $10^{7} / \mathrm{ml}$ in RPMI 1640 supplemented with $20 \%$ FBS and $10 \%$ DMSO. After rapid thawing, the cells were washed in complete RPMI 1640 medium containing 10\% heat-inactivated human serum (Sigma Chemical Co.), and tested for viability by trypan blue exclusion. Only samples with $\geq 80 \%$ viability were used. The antigens used were: recombinant gp110 protein and its deletion mutants with or without QKRAA, and peptides gp110-p, bo-p, and 11-p. Proliferation tests were performed in duplicate. Briefly, $5 \times 10^{5}$ cells per well in $200 \mu$ l of RPMI 1640 medium with $2 \mathrm{mM}$ glutamine, $10 \%$ heat-inactivated pooled human serum, $100 \mu \mathrm{g}$ $\mathrm{ml}^{-1}$ streptomycin, $100 \mathrm{U} \mathrm{ml}^{-1}$ penicillin, were cultured with the respective antigens, each at $10 \mu \mathrm{g} \mathrm{ml}^{-1}$, or with $1 \mu \mathrm{g} / \mathrm{ml} \mathrm{PHA}$. After $6 \mathrm{~d}$ at $37^{\circ} \mathrm{C}$ in $5 \% \mathrm{CO}_{2}$, cells were pulsed with $0.5 \mu \mathrm{Ci} \mathrm{ml}^{-1}\left[{ }^{3} \mathrm{H}\right]$ thymidine, harvested $16 \mathrm{~h}$ later on glass filters, and counted. The cpm of stimulated cells ranged between $3 \times 10^{4}$ and $1.8 \times 10^{5}$. Cell samples with a stimulation index $<3$ in response to PHA were excluded from analysis. All samples were tested blindly by the investigator.

Data analyses. Antibody specificity in RA patients was compared to that in healthy controls by the Wilcoxon rank sum test. The antibody levels and stimulation indexes of RA affected twins were compared to their unaffected twins with the Wilcoxon signed rank test. The intraclass correlation of antibody levels and stimulation indexes in twin pairs was computed via mixed effect ANOVA using a random effect for each pair of twins and a fixed effect for RA affected versus unaffected status. 


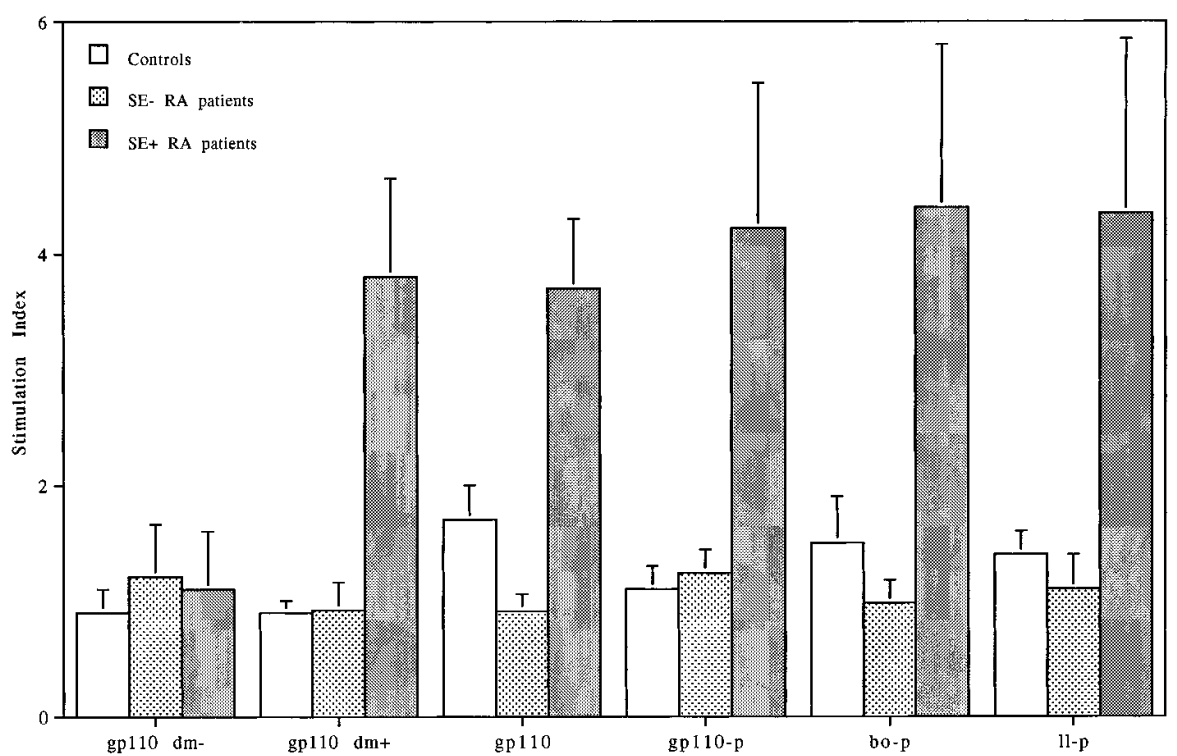

Figure 1. T cell proliferative responses in SE positive (+) RA patients $(n=25), \mathrm{SE}$ negative $(-)$ RA patients $(n=6)$, and control subjects $(n=14)$ to gp110, to its deletion mutants and to peptides gp110-p, bo-p, and ll-p. Tetanus toxoid was the positive control for both patients and controls. No significant differences between the three populations were found. cpm of stimulated cells ranged between $3 \times 10^{4}$ and $1.8 \times 10^{5}$.

\section{Results}

The SE is a T cell epitope in patients with RA. PBMC from SE positive patients with RA $(n=25)$ proliferated in response to recombinant gp110, to a deletion mutant which contains the QKRAA sequence, but not to the amino-terminal deletion mutant of gp110 not containing the shared epitope (Fig. 1). In contrast, PBMC from SE negative RA patients $(n=6)$ or healthy HLA-DRB1*0401 or *0101 positive controls $(n=14)$ did not proliferate to the QKRAA sequence, either as expressed on gp110 or on its carboxy-terminal deletion mutant. Only SE positive RA patients responded to gp110-p, ll-p, and bo-p peptides, which are derived from EBV, L. lactis dnaJ, and B. ovis dnaJ, respectively, and contain the RA susceptibility sequence (Fig. 1). Thus, the QKRAA sequence, even when derived from different microorganisms, elicits higher proliferative $\mathrm{T}$ cell responses in SE positive RA patients than in control subjects. Responses to PHA gave no significant differences between patients and controls. Range of responses to antigens was between $3 \times 10^{4}$ and $1.8 \times 10^{5} \mathrm{cpm}$. In addition, in six representative samples, $\mathrm{T}$ cell proliferation was partially inhibited by addition of either anti-DQ (Becton Dickinson Inc., Mountain View, CA) or anti-DR (Becton Dickinson) antibodies $\left(100 \mu \mathrm{g} \mathrm{ml}^{-1}\right)$.

The SE sequence is a dominant HLA class II binding motif. We performed peptide binding studies to ascertain whether $\mathrm{T}$ cell reactivity in RA patients could be ascribed to differences in QKRAA binding to HLA. Biotinylated gp110-p peptide bound equally well to HLA class II molecules extracted from a DRB1*0401 RA patient, from a matched HLA control, and from a DRB1*0701 normal individual (Table II). Also, biotinylated bo-p and ll-p peptides showed similar binding patterns to the three above described HLAs. No differences among subjects were found in HLA class II binding for self-p, an HLA-DRB1*0401-derived peptide containing the QKRAA sequence, the avidity of binding of which was about one-sixth that of gp110-p (Table II).

Epitope mapping shows that QKRAA is a target of specific humoral responses in $S E$ positive $R A$ patients. Significantly higher IgG titers against recombinant gp110 proteins contain- ing the QKRAA sequence were found in SE positive patients with RA when compared with SE negative patients, with normal controls, or with patients with SLE (Table III). gp110-p peptide inhibited antibody binding by $20.9 \%$ in the rheumatoids but only 5.3 or $6.9 \%$ in SE negative patients and healthy controls, respectively. It was therefore important to determine if the QKRAA sequence on two entirely different proteins, such as dnaJ and gp110, could display cross-reactivity. These studies were performed using sera from monozygotic twin pairs discordant for RA. Preincubation with dnaJ-p1 peptide inhibited antibody binding to gp110 more in the RA twins $(29 \pm 5.8 \%)$ than in the unaffected twins $(12.8 \pm 2.7 \%, P<$ 0.004) (Table IV). In a reciprocal manner, gp110-p peptide inhibited antibody binding to dnaJ more in the rheumatoids $(P<0.002)$.

$98 \%$ of the samples included in these studies had serological evidence of exposure to EBV, as assessed by antibodies to the EBV capsid antigen. Therefore, the responses in the RA patients are disease specific and not determined only by exposure to EBV.

ANOVA in monozygotic twin couples discordant for $R A$ suggests an influence from shared genetic or environmental factors in determining responses to $S E$ peptides. The above studies indicated that normal subjects do not have significant $\mathrm{T}$ cell responses to SE peptides. To analyze the possible effect of genetic background on immune responses to antigens containing the SE sequence, we evaluated 22 monozygotic twins for hu-

Table II. Binding of Peptides to HLA

\begin{tabular}{lllll}
\hline & \multicolumn{4}{c}{ Binding by biotinylated peptide } \\
\cline { 2 - 5 } \multicolumn{1}{c}{ HLA-DRB1 type } & gp110-p & bo-p & ll-p & self-p \\
\hline DRB1*0401 (patient) & 0.38 & 0.31 & 0.99 & 0.06 \\
DRB1*0401 (normal subject) & 0.36 & 0.33 & 0.95 & 0.07 \\
DRB1*0407 (normal subject) & 0.34 & 0.33 & 0.90 & 0.07 \\
& & & & \\
\hline
\end{tabular}

Arbitrary absorption units at $405 \mathrm{~nm}$. 
Table III. Specificity of Antibodies to EBV gp110 in RA Patients and Controls*

\begin{tabular}{|c|c|c|c|c|}
\hline Ab specificity & $\mathrm{SE}+\mathrm{RA}$ patients & SE- RA patients & Healthy controls & SLE \\
\hline & $n=73$ & $n=10$ & $n=56$ & $n=15$ \\
\hline Ab to gp110 deletion mutant with QKRAA & $150.7 \pm 12.1$ & $\begin{array}{c}114.1 \pm 21.0 \\
(<0.06)\end{array}$ & $\begin{array}{c}109.4 \pm 6.9 \\
(<0.001)\end{array}$ & $53.5 \pm 7$ \\
\hline Ab to whole gp110 & $131.1 \pm 5.2$ & $\begin{array}{l}98.1 \pm 10.5 \\
(<0.002)\end{array}$ & $\begin{array}{c}104.6 \pm 6.0 \\
(<0.005)\end{array}$ & ND \\
\hline Percent inhibition by gp110-p & $\begin{array}{c}20.9 \pm 4.4 \\
n=50\end{array}$ & $\begin{array}{r}5.3 \pm 3.9 \\
(<0.001)\end{array}$ & $\begin{array}{c}6.9 \pm 5.8 \\
n=36 \\
(<0.05)\end{array}$ & \\
\hline
\end{tabular}

*Values are expressed as percentage of standard \pm standard error at sera dilution 1:100. ND, Not done. $P$ values versus SE + patients are given in parentheses. Antibody titer to tetanus toxoid was comparable in all groups. Percent inhibition was assessed at gp110-p concentration of $100 \mu \mathrm{g} \mathrm{ml}^{-1}$, with serum dilution of 1:100.

moral responses to recombinant proteins dnaJ and gp110, and to recombinant deletion mutants of the same proteins containing the QKRAA sequence. All twin pairs were typed as HLADR4 positive. ANOVA performed on the twin set showed that common genetic or, perhaps, environmental agents significantly influence anti-dnaJ and anti-gp110 responses (Fig. 2, $A-D$, right-hand panels). The intraclass correlation was 0.67 for dnaJ, 0.78 for dnaJdm, 0.71 for gp110, and 0.64 for gp110dm. The coefficient is interpreted as the proportion of variance in each measurement due to genetic or shared environmental factors. Both healthy and affected twins had elevated titers of antibodies against $E$. coli dnaJ and EBV gp110 $(P=0.587$ for dnaJ and 0.176 for gp110), but only the affected twins showed higher specific response to dnaJdm and gp110dm $(P=0.002$ and 0.054 , respectively). As exposure to dnaJ or EBV is not sufficient to explain the presence of disease, the response to the SE in RA may be related to the etiopathogenetic mechanisms.

\section{Discussion}

The results of the experiments reported here show that SE positive patients with early RA have significantly high T lymphocyte proliferative responses to three different microbial antigens that express the QKRAA SE sequence. The SE positive RA patients' T cells responded to EBV gp110 and to dnaJ from B. ovis and L. lactis. Several experiments suggested that the QKRAA sequence was a major cross-reactive epitope on the three antigens. First, the patients' $\mathrm{T}$ cells responded strongly to deletion mutants which contained the QKRAA re-

Table IV. Inhibition of Antibody Binding to EBV gp110 and E. coli dnaJ in Monozygotic Twin Pairs $(n=14)$ Discordant for $R A$

\begin{tabular}{lccc}
\hline Percent binding inhibition & RA twins & & Healthy twins \\
\hline dnaJ by gp110-p & $31.6 \pm 3.6$ & $P<0.002$ & $10.1 \pm 1.9$ \\
gp110 by dnaJ-p1 & $29.0 \pm 5.8$ & $P<0.004$ & $12.8 \pm 2.7$
\end{tabular}

Values are expressed as percentage of standard \pm standard error at sera dilution of 1:100 and a peptide concentration of $100 \mu \mathrm{g} \mathrm{ml}^{-1}$. gion, but not to mutants that lacked it. Second, synthetic peptides encompassing the QKRAA region at their amino terminus stimulated $\mathrm{T}$ cell responses in RA patients. Third, cross-inhibition studies showed that only peptides containing QKRAA sequence could block antibody binding to the different recombinant antigens.

Previously, we showed that patients with early RA have abnormal cellular and humoral immune responses to the $E$. coli dnaJ protein which contains the QKRAA SE. One possible interpretation of the earlier experiments was that immune responses to the $E$. coli dnaJ were involved in RA pathogenesis (6). This conclusion must now be tempered with the current demonstration that several different organisms containing the QKRAA sequence are recognized by RA patients' $T$ cells. However, the mean stimulation index to the $E$. coli dnaJ epitope was about threefold higher than the other antigens, under conditions where $\mathrm{T}$ cell responses to tetanus toxoid and PHA were equivalent. Perhaps differences in affinity of the related $\mathrm{T}$ cell populations for the relevant MHC-peptide complexes or, more simply, different in vivo ligand concentrations may explain these results (7).

To address the issue whether the accentuated immune response to the QKRAA immunologic cassette was due to the presence of the SE in the HLA or to RA $(18,19)$, we assessed lymphocyte proliferation in SE negative RA patients and normal subjects typed as DRB1*0401 and DRB1*0404. We did not find any SE negative patient or normal subject with a PBL stimulation index $\geq 2.0$. We also evaluated in monozygotic twin pairs, discordant for the presence of RA, humoral responses to four recombinant antigens, all encompassing the QKRAA sequence. Twins affected by RA had higher titers of antibodies toward proteins expressing the SE than their healthy siblings. ANOVA in the two groups indicated that common genetic or environmental factors affect the magnitude of immune responses against proteins expressing QKRAA. The differences of immune repertoires, created by random rearrangements, may be responsible for differences of susceptibility to the disease. The analyses were confined to humoral responses because extensive prior studies performed by us and others revealed that normal subjects lacked detectable $\mathrm{T}$ cell responses to the SE in peripheral blood. In fact, PBL proliferative responses to commensal bacteria are uncommon in normal subjects, perhaps because the helper T cells for anti- 
A
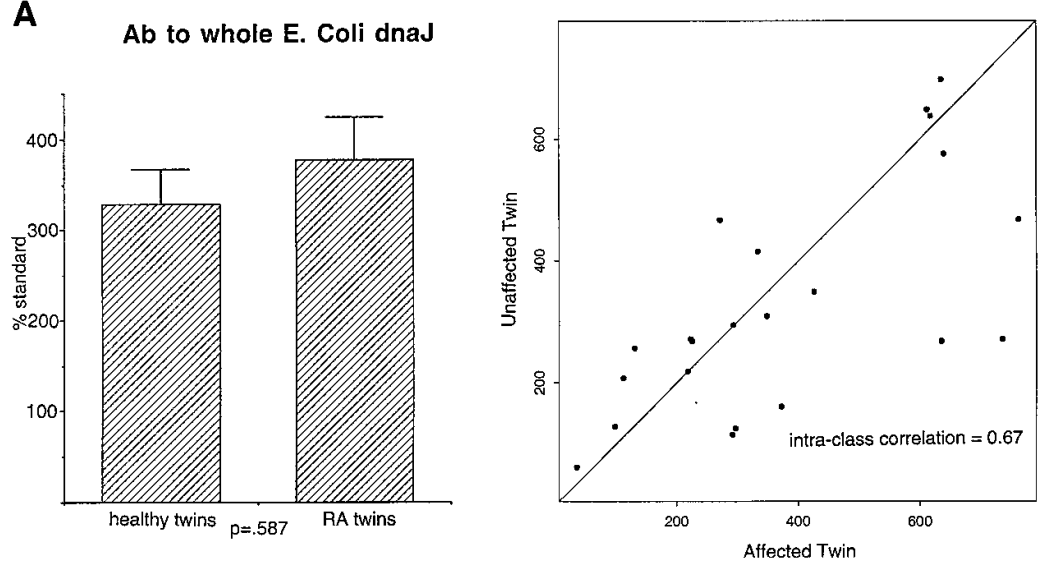

B

Ab to E. Coli dnaJ deletion mutant

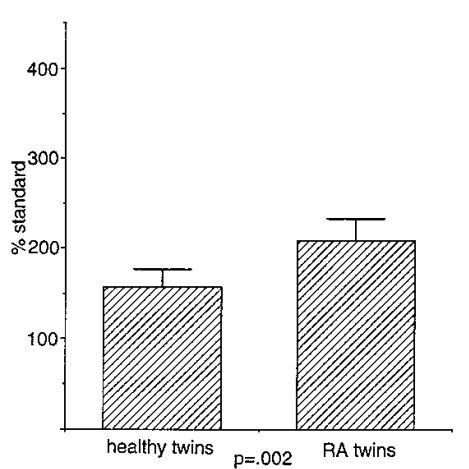

C Ab to whole EBV gp110

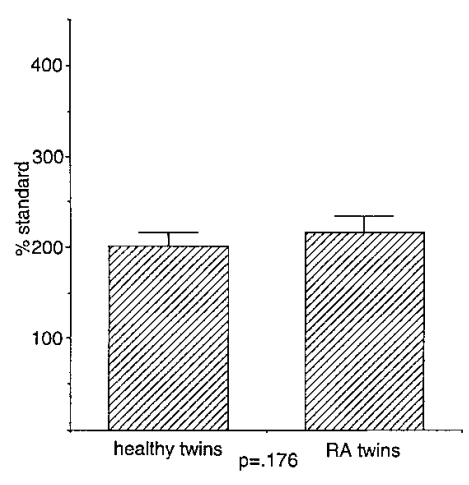

D

$A b$ to EBV gp110 deletion mutant

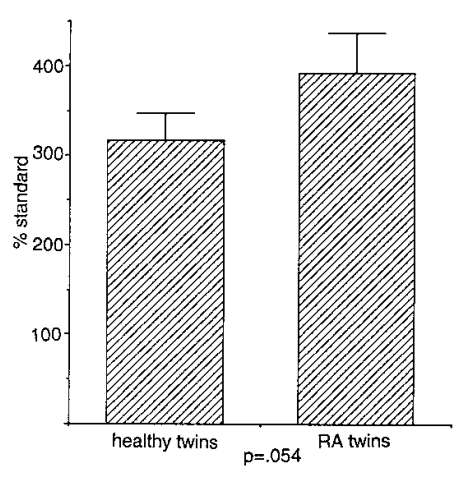

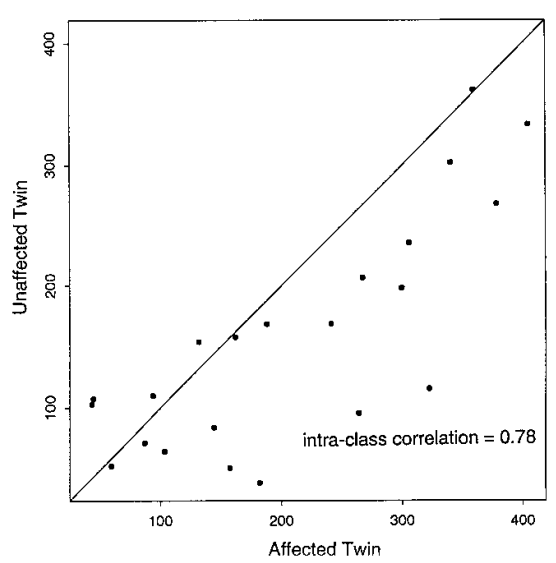
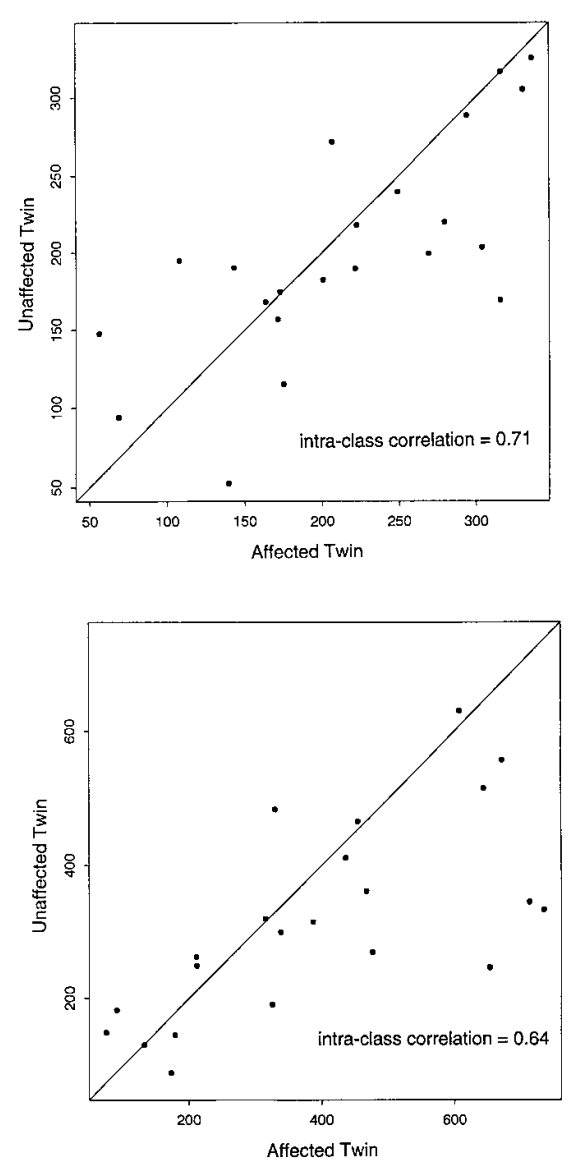

Figure 2. Specific IgG titers (left-hand panels), as determined by ELISA, of 22 patients with RA and their homozygous twins to dnaJ $(A)$, dnaJdm $(B)$, gp110 $(C)$, and gp110dm $(D)$. Results are expressed as the percentage of OD of a standard serum used in each test. Bars correspond to the means, error bars represent the standard errors. Right-hand figures for all panels indicate the intraclass correlation analysis for the respective antigens. Values for each sample are standardized, as described above. 
body production are confined to the gut-associated lymphoid tissues $(20,21)$. Binding studies have demonstrated the capability for antigen-presenting cells to present efficiently shared epitope peptides (this study and references 6,7). Therefore, failure to confine $\mathrm{T}$ cell responses against QKRAA epitope to mucosal tissues could represent an acquired immune abnormality in SE positive individuals which associates with the development of RA. In summary, the elevated peripheral blood T cells' proliferative responses to QKRAA of SE positive patients are specifically associated with rheumatoid disease, whereas the immune response genes influence magnitude and specificity of the antibody response to the epitope. The exact environmental factors which induce the peripheral spreading of the cellular immune response to the SE are not known (22, 23). The formal testing of this "multistep molecular mimicry" hypothesis may ultimately require the development of a better animal model of RA and analysis of the cytokine patterns of QKRAA-specific T cells. Future studies should also determine if the assessment of immune responses to one or several microbial antigens that express QKRAA has prognostic value in patients with early rheumatoid disease.

\section{Acknowledgments}

We wish to thank Ann C. Montemayor for providing HLA molecules for binding studies, Diane Amox for providing some RA patients' blood samples, Mrs. Nancy Noon for secretarial assistance, and Kabi Pharmacia Canada for their support of the Early Arthritis Research Program.

This study was supported in part by grants AR41897 and AR25443 from the National Institutes of Health. Salvatore Albani is an Investigator of the National Arthritis Research Foundation.

\section{References}

1. Statsny, P. 1978. Association of the B cell alloantigen DRw4 with rheumatoid arthritis. N. Engl. J. Med. 298:869-871.

2. Harris, E.D., Jr. 1990. Rheumatoid arthritis, pathophysiology and implications for therapy. N. Engl. J. Med. 322:1277-1289.

3. Ollier, W., and W. Thomson. 1992. Population genetics of rheumatoid arthritis. Rheum. Dis. Clin. N. Am. 18:741-761.

4. Albani, S., D.A. Carson, and J. Roudier. 1992. Genetic and environmental factors in the immune pathogenesis of rheumatoid arthritis. Rheum. Dis. Clin. N. Am., 18:729-740.

5. Gregersen, P., J. Silver, and R. Winchester. 1987. The shared epitope hypothesis. An approach to understand the molecular genetics of susceptibility to rheumatoid arthritis. Arthritis Rheum. 30:1205-1213.
6. Albani, S., E.C. Keystone, J.L. Nelson, W.E.R. Ollier, A. La Cava, A.C. Montemayor, D.A. Weber, C. Montecucco, A. Martini, and D.A. Carson. 1995 Positive selection in autoimmunity: abnormal immune responses to a bacterial dnaJ antigenic determinant in patients with early rheumatoid arthritis. Nat. Med. 1: 448-452.

7. Albani, S., and D.A. Carson. 1996. A multistep molecular mimicry hypothesis for the pathogenesis of rheumatoid arthritis. Immunol. Today. 17: 466470.

8. Baum, H., V. Brusic, K. Choudhuri, P. Cunningham, D. Vergani, and M. Peakman. 1995. Molecular mimicry in diabetes. Nat. Med. 1:388.

9. Solimena, M., and P. De Camilli. 1995. Coxsackievirus and diabetes. Nat. Med. 1:25-26.

10. Roudier, J., G. Rhodes, J. Petersen, J. Vaughan, and D.A. Carson. 1988. The Epstein Barr virus glycoprotein gp110, a molecular link between HLA DR4, HLA DR1 and rheumatoid arthritis. Scand. J. Immunol. 27:367-371.

11. Roudier, J., J. Petersen, G.H. Rhodes, J. Luka, and D.A. Carson. 1989. Susceptibility to rheumatoid arthritis maps to a T-cell epitope shared by the HLA-Dw4 DR $\beta$-1 chain and the Epstein-Barr virus glycoprotein gp110. Proc. Natl. Acad. Sci. USA. 86:5104-5108.

12. Albani, S., J.E. Tuckwell, L. Esparza, D.A. Carson, and J. Roudier. 1992. The susceptibility sequence to rheumatoid arthritis is a cross-reactive B cell epitope shared by Escherichia coli heat shock protein dnaJ and the histocompatibility leukocyte antigen DRB1*0401 molecule. J. Clin. Invest. 89:327331.

13. Bardwell, J.C., K. Tilly, E. Craig, J. King, M. Zylic, and C. Georgopoulos. 1986. The nucleotide sequence of the Escherichia coli $\mathrm{K} 12$ dnaJ+ gene. A gene that encodes a heat shock protein. J. Biol. Chem. 261:1782-1785.

14. Cellier, M.F., J. Teyssier, M. Nicolas, J.P. Liautard, J. Marti, and J. Sri Widada. 1992. Cloning and characterization of the Brucella ovis heat shock protein DnaK functionally expressed in Escherichia coli. J. Bacteriol. 174:80368042 .

15. Van Asseldork, M., M. Simons, M. Visser, W.M. de Vos, and G. Simons. 1993. Cloning nucleotide sequence and regulatory analysis of the Lactococcus lactis dnaJ gene. J. Bacteriol. 175:1637-1644.

16. Gough, A., J. Faint, M. Salmon, A. Hassel, P. Wordsworth, D. Pilling, A. Birley, and P. Emery. 1994. Genetic typing of patients with inflammatory arthritis at presentation can be used to predict outcome. Arthritis Rheum. 37: 1166-1170.

17. Chicz, R.M., R.G. Urban, W.S. Lane, J.C. Gorga, L.J. Stern, D.A. Vignali, and J.L. Strominger. 1992. Predominant naturally processed peptides bound to HLA-DR1 are derived from MHC-related molecules and are heterogeneous in size. Nature (Lond.). 358:764-768.

18. Weyand, C.M., T.G. McCarthy, and J.J. Goronzy. 1995. Correlation between disease phenotype and genetic heterogeneity in rheumatoid arthritis. $J$. Clin. Invest. 95:2120-2126.

19. Li, Y., G.R. Sun, J.R. Tumang, M.K. Crow, and S.M. Friedman. 1994 CDR3 sequence motifs shared by oligoclonal rheumatoid arthritis synovial $\mathrm{T}$ cells. Evidence for an antigen-driven response. J. Clin. Invest. 94:2525-2531.

20. Pirzer, U.C., G. Schurmann, S. Post, M. Betzler, and S.C. Meuer. 1990. Differential responsiveness to CD3-Ti vs. CD2-dependent activation of human intestinal T lymphocytes. Eur. J. Immunol. 20:2339-2342.

21. Skoldstam, L., and K.E. Magnusson. 1991. Fasting, intestinal permeability, and rheumatoid arthritis. Rheum. Dis. Clin. N. Am. 17:363-371.

22. Winfield, J.B. 1989. Stress proteins, arthritis, and autoimmunity. Arthritis Rheum. 32:1497-1504.

23. Zanelli, E., M.A. Gonzalez-Gay, and C.S. David. 1995. Could HLADRB1 be the protective locus in rheumatoid arthritis? Immunol. Today. 16: 274-278. 